\title{
Higher Tetrahedral Algebras
}

\author{
Karin Erdmann ${ }^{1}$ Andrzej Skowroński²
}

Received: 27 June 2017 / Accepted: 7 February 2018 / Published online: 20 February 2018

(C) The Author(s) 2018. This article is an open access publication

\begin{abstract}
We introduce and study the higher tetrahedral algebras, an exotic family of finitedimensional tame symmetric algebras over an algebraically closed field. The Gabriel quiver of such an algebra is the triangulation quiver associated to the coherent orientation of the tetrahedron. Surprisingly, these algebras occurred in the classification of all algebras of generalized quaternion type, but are not weighted surface algebras. We prove that a higher tetrahedral algebra is periodic if and only if it is non-singular.
\end{abstract}

Keywords Syzygy $\cdot$ Periodic algebra $\cdot$ Symmetric algebra $\cdot$ Tame algebra

Mathematics Subject Classification (2010) $16 \mathrm{D} 50 \cdot 16 \mathrm{G} 20 \cdot 16 \mathrm{G} 60 \cdot 16 \mathrm{~S} 80$

\section{Introduction and the Main Results}

Throughout this paper, $K$ will denote a fixed algebraically closed field. By an algebra we mean an associative finite-dimensional $K$-algebra with an identity. For an algebra $A$, we

Presented by Jon F. Carlson.

The research was supported by the research grant DEC-2011/02/A/ST1/00216 of the National Science Center Poland.

Andrzej Skowroński

skowron@mat.uni.torun.pl

Karin Erdmann

erdmann@maths.ox.ac.uk

1 Mathematical Institute, Oxford University, ROQ, Oxford OX2 6GG, UK

2 Faculty of Mathematics and Computer Science, Nicolaus Copernicus University, Chopina 12/18, 87-100 Toruń, Poland 
denote by $\bmod A$ the category of finite-dimensional right $A$-modules and by $D$ the standard duality $\operatorname{Hom}_{K}(-, K)$ on $\bmod A$. An algebra $A$ is called self-injective if $A_{A}$ is injective in $\bmod A$, or equivalently, the projective modules in $\bmod A$ are injective. A prominent class of self-injective algebras is formed by the symmetric algebras $A$ for which there exists an associative, non-degenerate symmetric $K$-bilinear form $(-,-): A \times A \rightarrow K$. Classical examples of symmetric algebras are provided by the blocks of group algebras of finite groups and the Hecke algebras of finite Coxeter groups. In fact, any algebra $A$ is a quotient algebra of its trivial extension algebra $\mathrm{T}(A)=A \ltimes D(A)$, which is a symmetric algebra.

From the remarkable Tame and Wild Theorem of Drozd (see $[4,8])$ the class of algebras over $K$ may be divided into two disjoint classes. The first class consists of the tame algebras for which the indecomposable modules occur in each dimension $d$ in a finite number of discrete and a finite number of one-parameter families. The second class is formed by the wild algebras whose representation theory comprises the representation theories of all algebras over $K$. Accordingly, we may realistically hope to classify the indecomposable finite-dimensional modules only for the tame algebras. Among the tame algebras we may distinguish the algebras of polynomial growth for which the number of one-parameter families of indecomposable modules in each dimension $d$ is bounded by $d^{m}$ for some positive integer $m$ (depending only on the algebra) whose representation theory is usually well understood (see $[2,23,24]$ for some general results). On the other hand, the representation theory of tame algebras of non-polynomial growth is still only emerging.

Let $A$ be an algebra. Given a module $M$ in $\bmod A$, its syzygy is defined to be the kernel $\Omega_{A}(M)$ of a minimal projective cover of $M$ in $\bmod A$. The syzygy operator $\Omega_{A}$ is a very important tool to construct modules in $\bmod A$ and relate them. For $A$ self-injective, it induces an equivalence of the stable module category $\underline{\bmod } A$, and its inverse is the shift of a triangulated structure on $\bmod A$ [15]. A module $M$ in $\bmod A$ is said to be periodic if $\Omega_{A}^{n}(M) \cong M$ for some $n \geq 1$, and if so the minimal such $n$ is called the period of $M$. The action of $\Omega_{A}$ on $\bmod A$ can effect the algebra structure of $A$. For example, if all simple modules in $\bmod A$ are periodic, then $A$ is a self-injective algebra. An algebra $A$ is defined to be periodic if it is periodic viewed as a module over the enveloping algebra $A^{e}=A^{\mathrm{op}} \otimes_{K} A$, or equivalently, as an $A$ - $A$-bimodule. It is known that if $A$ is a periodic algebra of period $n$ then for any indecomposable non-projective module $M$ in $\bmod A$ the syzygy $\Omega_{A}^{n}(M)$ is isomorphic to $M$ (see [26, Theorem IV.11.19]).

Finding or possibly classifying periodic algebras is an important problem. It is very interesting because of connections with group theory, topology, singularity theory and cluster algebras. Periodicity of an algebra, and its period, are invariant under derived equivalences [20] (see also [10]). Therefore, to study periodic algebras we may assume that the algebras are basic and indecomposable.

We are concerned with the classification of all periodic tame symmetric algebras. In [9] Dugas proved that every representation-finite self-injective algebra, without simple blocks, is a periodic algebra. We note that, by general theory (see [24, Section 3]), a basic, indecomposable, non-simple, symmetric algebra $A$ is representation-finite if and only if $A$ is socle equivalent to an algebra $\mathrm{T}(B)^{G}$ of invariants of the trivial extension algebra $\mathrm{T}(B)$ of a tilted algebra $B$ of Dynkin type with respect to free action of a finite cyclic group $G$. The representation-infinite, indecomposable, periodic algebras of polynomial growth were classified by Białkowski, Erdmann and Skowroński in [2] (see also [23, 24]). In particular, it follows from [2] that every basic, indecomposable, periodic, representation-infinite symmetric tame algebra of polynomial growth is socle equivalent to an algebra $\mathrm{T}(B)^{G}$ of invariants of the trivial extension algebra $\mathrm{T}(B)$ of a tubular algebra $B$ of tubular type 
$(2,2,2,2),(3,3,3),(2,4,4),(2,3,6)$ (introduced by Ringel [21]) with respect to free action of a finite cyclic group $G$.

Recently we introduced in [11] the weighted surface algebras of triangulated surfaces with arbitrarily oriented triangles and proved that all these algebras, except the singular tetrahedral algebras, are periodic tame symmetric algebras of period 4 . Here, we investigate the periodicity of higher tetrahedral algebras, being "higher analogs" of the tetrahedral algebras studied in [11]. These algebras occurred in the authors work concerning the classification of all representation-infinite tame symmetric periodic algebras of period 4 .

Consider the tetrahedron

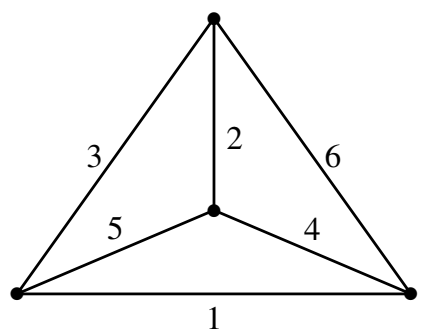

with the coherent orientation of triangles: (1 5 4), (2 5 3), (2 64 ), (1 6 3). Then, following [11], we have the associated triangulation quiver $(Q, f)$ of the form

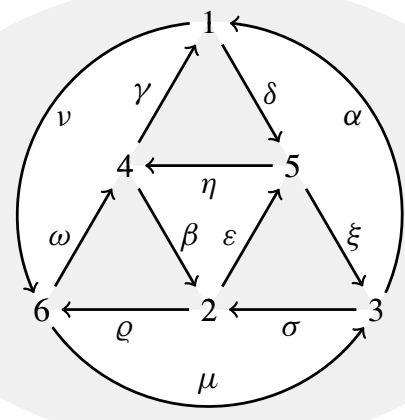

where $f$ is the permutation of arrows of order 3 described by the shaded subquivers. We denote by $g$ the permutation on the set of arrows of $Q$ whose $g$-orbits are the four white 3-cycles.

Let $m \geq 1$ be a natural number and $\lambda \in K$. We denote by $\Lambda(m, \lambda)$ the algebra given by the above quiver and the relations:

$$
\begin{aligned}
& \gamma \delta=\beta \varepsilon+\lambda(\beta \varrho \omega)^{m-1} \beta \varepsilon, \quad \delta \eta=\nu \omega, \quad \eta \gamma=\xi \alpha, \quad \nu \mu=\delta \xi, \\
& \varrho \omega=\varepsilon \eta+\lambda(\varepsilon \xi \sigma)^{m-1} \varepsilon \eta, \quad \omega \beta=\mu \sigma, \quad \beta \varrho=\gamma \nu, \quad \mu \alpha=\omega \gamma, \\
& \xi \sigma=\eta \beta+\lambda(\eta \gamma \delta)^{m-1} \eta \beta, \quad \sigma \varepsilon=\alpha \delta, \quad \varepsilon \xi=\varrho \mu, \quad \alpha \nu=\sigma \varrho, \\
& \left(\theta f(\theta) f^{2}(\theta)\right)^{m-1} \theta f(\theta) g(f(\theta))=0 \text { for any arrow } \theta \text { in } Q \text {. }
\end{aligned}
$$

We call $\Lambda(m, \lambda)$ a tetrahedral algebra of degree $m$. Moreover, an algebra $\Lambda(m, \lambda)$ with $\lambda \in K^{*}=K \backslash\{0\}$ is said to be a non-singular tetrahedral algebra of degree $m$. We note that for $m=1, \Lambda(1, \lambda)$ is the tetrahedral algebra $\Lambda(\mathbb{S}, \lambda+1)$ investigated in [11, Section 6$]$, 
and is a weighted surface algebra. Further, the algebra $\Lambda(1,0)=\Lambda(\mathbb{S}, 1)$ is called in [11] the singular tetrahedral algebra, because it is a unique algebra among all weighted surface algebras without periodic simple modules. On the other hand, a non-singular tetrahedral algebra $\Lambda(1, \lambda), \lambda \in K^{*}$, is a periodic algebra of period 4, with all simple modules being periodic of period 4 .

We call a tetrahedral algebra $\Lambda(m, \lambda)$ of degree $m \geq 2$ a higher tetrahedral algebra. The aim of this article is to describe basic properties of the higher tetrahedral algebras, which extend results on the tetrahedral algebras (of degree 1), established in [11], to the algebras given by the same quiver but much more complicated higher degree relations.

The following two theorems describe some properties of higher tetrahedral algebras.

Theorem 1 Let $\Lambda=\Lambda(m, \lambda)$ be a higher tetrahedral algebra. Then $\Lambda$ is a finitedimensional symmetric algebra with $\operatorname{dim}_{K} \Lambda=36 \mathrm{~m}$.

Theorem 2 Let $\Lambda=\Lambda(m, \lambda)$ be a higher tetrahedral algebra. Then $\Lambda$ is a tame algebra of non-polynomial growth.

The following theorem is the main result of the paper.

Theorem 3 Let $\Lambda=\Lambda(m, \lambda)$ be a higher tetrahedral algebra. Then the following statements are equivalent:

(i) $\bmod \Lambda$ admits a periodic simple module.

(ii) All simple modules in $\bmod \Lambda$ are periodic of period 4.

(iii) $\Lambda$ is a periodic algebra of period 4.

(iv) $\Lambda$ is non-singular.

Following [12], an algebra $A$ is called an algebra of generalized quaternion type if $A$ is representation-infinite tame symmetric and every simple module in $\bmod A$ is periodic of period 4. Theorem 3 is one of the essential ingredients for proving in [12, Main Theorem] that an algebra $A$ is of generalized quaternion type with 2-regular Gabriel quiver if and only if $A$ is a socle deformation of a weighted surface algebra, different from the singular tetrahedral algebra, or is a non-singular higher tetrahedral algebra. On the other hand, it is expected that the singular higher tetrahedral algebras $\Lambda(m, 0), m \geq 2$, will form an exotic family of algebras of generalized semidihedral type.

This paper is organized as follows. In Section 2 we recall background on special biserial algebras and degenerations of algebras. In Section 3 we describe our general approach and results for constructing a minimal projective bimodule resolution of an algebra with periodic simple modules. Section 4 is devoted to basic properties of the higher tetrahedral algebras and the proof of Theorem 1. Sections 5 and 6 contain the proofs of Theorems 2 and 3, respectively.

For general background on the relevant representation theory we refer to the books [1, $22,26]$.

\section{Preliminary Results}

A quiver is a quadruple $Q=\left(Q_{0}, Q_{1}, s, t\right)$ consisting of a finite set $Q_{0}$ of vertices, a finite set $Q_{1}$ of arrows, and two maps $s, t: Q_{1} \rightarrow Q_{0}$ which associate to each arrow $\alpha \in Q_{1}$ 
its source $s(\alpha) \in Q_{0}$ and its target $t(\alpha) \in Q_{0}$. We denote by $K Q$ the path algebra of $Q$ over $K$ whose underlying $K$-vector space has as its basis the set of all paths in $Q$ of length $\geq 0$, and by $R_{Q}$ the arrow ideal of $K Q$ generated by all paths in $Q$ of length $\geq 1$. An ideal $I$ in $K Q$ is said to be admissible if there exists $m \geq 2$ such that $R_{Q}^{m} \subseteq I \subseteq R_{Q}^{2}$. If $I$ is an admissible ideal in $K Q$, then the quotient algebra $K Q / I$ is called a bound quiver algebra, and is a finite-dimensional basic $K$-algebra. Moreover, $K Q / I$ is indecomposable if and only if $Q$ is connected. Every basic, indecomposable, finite-dimensional $K$-algebra $A$ has a bound quiver presentation $A \cong K Q / I$, where $Q=Q_{A}$ is the Gabriel quiver of $A$ and $I$ is an admissible ideal in $K Q$. For a bound quiver algebra $A=K Q / I$, we denote by $e_{i}, i \in Q_{0}$, the associated complete set of pairwise orthogonal primitive idempotents of $A$, and by $S_{i}=e_{i} A / e_{i} \operatorname{rad} A$ (respectively, $P_{i}=e_{i} A$ ), $i \in Q_{0}$, the associated complete family of pairwise non-isomorphic simple modules (respectively, indecomposable projective modules) in $\bmod A$.

Following [25], an algebra $A$ is said to be special biserial if $A$ is isomorphic to a bound quiver algebra $K Q / I$, where the bound quiver $(Q, I)$ satisfies the following conditions:

(a) each vertex of $Q$ is a source and target of at most two arrows,

(b) for any arrow $\alpha$ in $Q$ there are at most one arrow $\beta$ and at most one arrow $\gamma$ with $\alpha \beta \notin I$ and $\gamma \alpha \notin I$.

Moreover, if in addition $I$ is generated by paths of $Q$, then $A=K Q / I$ is said to be a string algebra [3]. It was proved in [19] that the class of special biserial algebras coincides with the class of biserial algebras (indecomposable projective modules have biserial structure) which admit simply connected Galois coverings. Furthermore, by [27, Theorem 1.4] we know that every special biserial algebra is a quotient algebra of a symmetric special biserial algebra. We also mention that, if $A$ is a self-injective special biserial algebra, then $A / \operatorname{soc}(A)$ is a string algebra.

The following has been proved by Wald and Waschbüsch in [27] (see also [3, 7] for alternative proofs).

\section{Proposition 2.1 Every special biserial algebra is tame.}

For a positive integer $d$, we denote by $\operatorname{alg}_{d}(K)$ the affine variety of associative $K$-algebra structures with identity on the affine space $K^{d}$. Then the general linear group $\mathrm{GL}_{d}(K)$ acts on $\operatorname{alg}_{d}(K)$ by transport of the structures, and the $\mathrm{GL}_{d}(K)$-orbits in $\operatorname{alg}_{d}(K)$ correspond to the isomorphism classes of $d$-dimensional algebras (see [17] for details). We identify a $d$-dimensional algebra $A$ with the point of $\operatorname{alg}_{d}(K)$ corresponding to it. For two $d$-dimensional algebras $A$ and $B$, we say that $B$ is a degeneration of $A$ ( $A$ is a deformation of $B$ ) if $B$ belongs to the closure of the $\mathrm{GL}_{d}(K)$-orbit of $A$ in the Zariski topology of $\operatorname{alg}_{d}(K)$.

Geiss' Theorem [13] shows that if $A$ and $B$ are two $d$-dimensional algebras, $A$ degenerates to $B$ and $B$ is a tame algebra, then $A$ is also a tame algebra (see also [5]). We will apply this theorem in the following special situation.

Proposition 2.2 Let $d$ be a positive integer, and $A(t), t \in K$, be an algebraic family in $\operatorname{alg}_{d}(K)$ such that $A(t) \cong A(1)$ for all $t \in K \backslash\{0\}$. Then $A(1)$ degenerates to $A(0)$. In particular, if $A(0)$ is tame, then $A(1)$ is tame.

A family of algebras $A(t), t \in K$, in $\operatorname{alg}_{d}(K)$ is said to be algebraic if the induced map $A(-): K \rightarrow \operatorname{alg}_{d}(K)$ is a regular map of affine varieties. 


\section{Bimodule Resolutions of Self-Injective Algebras}

In this section we describe a general approach for proving that an algebra $A$ with periodic simple modules is a periodic algebra.

Let $A=K Q / I$ be a bound quiver algebra, and $e_{i}, i \in Q_{0}$, be the primitive idempotents of $A$ associated to the vertices of $Q$. Then $e_{i} \otimes e_{j}, i, j \in Q_{0}$, form a set of pairwise orthogonal primitive idempotents of the enveloping algebra $A^{e}=A^{\mathrm{op}} \otimes_{K} A$ whose sum is the identity of $A^{e}$. Hence, $P(i, j)=\left(e_{i} \otimes e_{j}\right) A^{e}=A e_{i} \otimes e_{j} A$, for $i, j \in Q_{0}$, form a complete set of pairwise non-isomorphic indecomposable projective modules in $\bmod A^{e}$ (see [26, Proposition IV.11.3]).

The following result by Happel [16, Lemma 1.5] describes the terms of a minimal projective resolution of $A$ in $\bmod A^{e}$.

Proposition 3.1 Let $A=K Q / I$ be a bound quiver algebra. Then there is in $\bmod A^{e} a$ minimal projective resolution of $A$ of the form

$$
\cdots \rightarrow \mathbb{P}_{n} \stackrel{d_{n}}{\rightarrow} \mathbb{P}_{n-1} \rightarrow \cdots \rightarrow \mathbb{P}_{1} \stackrel{d_{1}}{\rightarrow} \mathbb{P}_{0} \stackrel{d_{0}}{\rightarrow} A \rightarrow 0,
$$

where

$$
\mathbb{P}_{n}=\bigoplus_{i, j \in Q_{0}} P(i, j)^{\operatorname{dim}_{K} \operatorname{Ext}_{A}^{n}\left(S_{i}, S_{j}\right)}
$$

for any $n \in \mathbb{N}$

The syzygy modules have an important property, a proof for the next Lemma may be found in [26, Lemma IV.11.16].

Lemma 3.2 Let $A$ be an algebra. For any positive integer $n$, the module $\Omega_{A^{e}}^{n}(A)$ is projective as a left A-module and also as a right A-module.

There is no general recipe for the differentials $d_{n}$ in Proposition 3.1, except for the first three which we will now describe. This will be one of the main techniques we use in our proof of the implication (iv) $\Rightarrow$ (iii) in Theorem 3 .

We have

$$
\mathbb{P}_{0}=\bigoplus_{i \in Q_{0}} P(i, i)=\bigoplus_{i \in Q_{0}} A e_{i} \otimes e_{i} A
$$

The homomorphism $d_{0}: \mathbb{P}_{0} \rightarrow A$ in $\bmod A^{e}$ defined by $d_{0}\left(e_{i} \otimes e_{i}\right)=e_{i}$ for all $i \in Q_{0}$ is a minimal projective cover of $A$ in $\bmod A^{e}$. Recall that, for two vertices $i$ and $j$ in $Q$, the number of arrows from $i$ to $j$ in $Q$ is equal to $\operatorname{dim}_{K} \operatorname{Ext}_{A}^{1}\left(S_{i}, S_{j}\right)$ (see [1, Lemma III.2.12]). Hence we have

$$
\mathbb{P}_{1}=\bigoplus_{\alpha \in Q_{1}} P(s(\alpha), t(\alpha))=\bigoplus_{\alpha \in Q_{1}} A e_{s(\alpha)} \otimes e_{t(\alpha)} A
$$

Then we have the following known fact (see [2, Lemma 3.3] for a proof).

Lemma 3.3 Let $A=K Q / I$ be a bound quiver algebra, and $d_{1}: \mathbb{P}_{1} \rightarrow \mathbb{P}_{0}$ the homomorphism in $\bmod A^{e}$ defined by

$$
d_{1}\left(e_{s(\alpha)} \otimes e_{t(\alpha)}\right)=\alpha \otimes e_{t(\alpha)}-e_{s(\alpha)} \otimes \alpha
$$

for any arrow $\alpha$ in $Q$. Then $d_{1}$ induces a minimal projective cover $d_{1}: \mathbb{P}_{1} \rightarrow \Omega_{A^{e}}^{1}(A)$ of $\Omega_{A^{e}}^{1}(A)=\operatorname{Ker} d_{0}$ in $\bmod A^{e}$. In particular, we have $\Omega_{A^{e}}^{2}(A) \cong \operatorname{Ker} d_{1}$ in $\bmod A^{e}$. 
We will denote the homomorphism $d_{1}: \mathbb{P}_{1} \rightarrow \mathbb{P}_{0}$ by $d$. For the algebras $A$ we will consider, the kernel $\Omega_{A^{e}}^{2}(A)$ of $d$ will be generated, as an $A$ - $A$-bimodule, by some elements of $\mathbb{P}_{1}$ associated to a set of relations generating the admissible ideal $I$. Recall that a relation in the path algebra $K Q$ is an element of the form

$$
\mu=\sum_{r=1}^{n} c_{r} \mu_{r}
$$

where $c_{1}, \ldots, c_{r}$ are non-zero elements of $K$ and $\mu_{r}=\alpha_{1}^{(r)} \alpha_{2}^{(r)} \ldots \alpha_{m_{r}}^{(r)}$ are paths in $Q$ of length $m_{r} \geq 2, r \in\{1, \ldots, n\}$, having a common source and a common target. The admissible ideal $I$ can be generated by a finite set of relations in $K Q$ (see [1, Corollary II.2.9]). In particular, the bound quiver algebra $A=K Q / I$ is given by the path algebra $K Q$ and a finite number of identities $\sum_{r=1}^{n} c_{r} \mu_{r}=0$ given by a finite set of generators of the ideal $I$. Consider the $K$-linear homomorphism $\pi: K Q \rightarrow \mathbb{P}_{1}$ which assigns to a path $\alpha_{1} \alpha_{2} \ldots \alpha_{m}$ in $Q$ the element

$$
\pi\left(\alpha_{1} \alpha_{2} \ldots \alpha_{m}\right)=\sum_{k=1}^{m} \alpha_{1} \alpha_{2} \ldots \alpha_{k-1} \otimes \alpha_{k+1} \ldots \alpha_{m}
$$

in $\mathbb{P}_{1}$, where $\alpha_{0}=e_{s\left(\alpha_{1}\right)}$ and $\alpha_{m+1}=e_{t\left(\alpha_{m}\right)}$. Observe that $\pi\left(\alpha_{1} \alpha_{2} \ldots \alpha_{m}\right) \in e_{s\left(\alpha_{1}\right)} \mathbb{P}_{1} e_{t\left(\alpha_{m}\right)}$. Then, for a relation $\mu=\sum_{r=1}^{n} c_{r} \mu_{r}$ in $K Q$ lying in $I$, we have an element

$$
\pi(\mu)=\sum_{r=1}^{n} c_{r} \pi\left(\mu_{r}\right) \in e_{i} \mathbb{P}_{1} e_{j}
$$

where $i$ is the common source and $j$ is the common target of the paths $\mu_{1}, \ldots, \mu_{r}$. The following lemma shows that relations always produce elements in the kernel of $d_{1}$; the proof is straightforward.

Lemma 3.4 Let $A=K Q / I$ be a bound quiver algebra and $d_{1}: \mathbb{P}_{1} \rightarrow \mathbb{P}_{0}$ the homomorphism in $\bmod A^{e}$ defined in Lemma 3.3. Then for any relation $\mu$ in $K Q$ lying in $I$, we have $d_{1}(\pi(\mu))=0$.

For an algebra $A=K Q / I$ in our context, we will see that there exists a family of relations $\mu^{(1)}, \ldots, \mu^{(q)}$ generating the ideal $I$ such that the associated elements $\pi\left(\mu^{(1)}\right), \ldots, \pi\left(\mu^{(q)}\right)$ generate the $A$-A-bimodule $\Omega_{A^{e}}^{2}(A)=\operatorname{Ker} d_{1}$. In fact, using Lemma 3.2, we will be able to show that

$$
\mathbb{P}_{2}=\bigoplus_{j=1}^{q} P\left(s\left(\mu^{(j)}\right), t\left(\mu^{(j)}\right)\right)=\bigoplus_{j=1}^{q} A e_{s\left(\mu^{(j)}\right)} \otimes e_{t\left(\mu^{(j)}\right)} A,
$$

and the homomorphism $d_{2}: \mathbb{P}_{2} \rightarrow \mathbb{P}_{1}$ in $\bmod A^{e}$ such that

$$
d_{2}\left(e_{s\left(\mu^{(j)}\right)} \otimes e_{t\left(\mu^{(j)}\right)}\right)=\pi\left(\mu^{(j)}\right),
$$

for $j \in\{1, \ldots, q\}$, defines a projective cover of $\Omega_{A^{e}}^{2}(A)$ in mod $A^{e}$. In particular, we have $\Omega_{A^{e}}^{3}(A) \cong \operatorname{Ker} d_{2}$ in $\bmod A^{e}$. We will denote this homomorphism $d_{2}$ by $R$.

For the next map $d_{3}: \mathbb{P}_{3} \rightarrow \mathbb{P}_{2}$, which we will call $S:=d_{3}$ later, we do not have a general recipe. To define it, we need a set of minimal generators for $\Omega_{A^{e}}^{3}(A)$, and Proposition 3.1 tells us where we should look for them. 


\section{Proof of Theorem 1}

Let $\Lambda=\Lambda(m, \lambda)$ for some $m \geq 2$ and $\lambda \in K$. In this section we will study algebra properties of $\Lambda$, and in particular prove Theorem 1 . The first results will be used to reduce calculations, and should also be of independent interest.

In order to construct a basis of $\Lambda$ with good properties, we analyze the images of paths in $\Lambda$, they have very unusual properties. We introduce some notation. It follows from the relations defining $\Lambda$ that we may define the elements

$$
\begin{array}{lll}
X_{1}=\delta \eta \gamma=v \mu \alpha, & X_{2}=\varrho \omega \beta=\varepsilon \xi \sigma, & X_{3}=\alpha \nu \mu=\sigma \varepsilon \xi, \\
X_{4}=\gamma \delta \eta=\beta \varrho \omega, & X_{5}=\eta \gamma \delta=\xi \sigma \varepsilon, & X_{6}=\omega \beta \varrho=\mu \alpha \nu,
\end{array}
$$

given by products of the arrows around the shaded triangles. Moreover, we define the elements

$$
\tilde{X}_{2}=\varepsilon \eta \beta, \quad \tilde{X}_{4}=\beta \varepsilon \eta, \quad \tilde{X}_{5}=\eta \beta \varepsilon .
$$

The quiver $Q$ of $\Lambda$ has an automorphism $\varphi$ of order 3, defined as follows. Its action on vertices is given by the cycles

$$
\left(\begin{array}{lllll}
5 & 4 & 2
\end{array}\right)\left(\begin{array}{llll}
1 & 6 & 3
\end{array}\right)
$$

and the action on arrows is

$$
(\delta \omega \sigma)(\eta \beta \varepsilon)(\gamma \rho \xi)(v \mu \alpha) \text {. }
$$

Lemma 4.1 The action of $\varphi$ extends to an algebra automorphism of $\Lambda$.

Proof We extend $\varphi$ to an algebra map of $K Q$. Then we must check that $\varphi$ preserves the relations, which is direct calculation. For example,

$$
\varphi(\gamma \delta)=\varphi(\gamma) \varphi(\delta)=\rho \omega \text { and } \varphi(\beta \varepsilon)=\varphi(\beta) \varphi(\varepsilon)=\varepsilon \eta,
$$

and

$$
\varphi\left(X_{4}\right)=\varphi(\gamma) \varphi(\delta) \varphi(\eta)=\rho \omega \beta=X_{2} .
$$

Hence, $\varphi$ takes the relation for $\gamma \delta$ to the relation for $\rho \omega$.

Lemma 4.2 For each vertex $i$ of $Q$, the element $X_{i}^{m}$ belongs to the right socle of $\Lambda$.

Proof It follows from the relations that, for each arrow $\theta$ in $Q$, we have $X_{s(\theta)}^{m} \theta=0$. For example, we have

$$
X_{1}^{m} \delta=(\nu \mu \alpha)^{m} \delta=v(\mu \alpha v)^{m-1} \mu \alpha \delta=v\left(X_{s(\mu)}\right)^{m-1} \mu f(\mu) g(f(\mu))=0 .
$$

Lemma 4.3 We have the following equalities in $\Lambda$.

(i) $X_{1}=v \omega \gamma=\delta \xi \alpha, X_{3}=\sigma \varrho \mu=\alpha \delta \xi, X_{6}=\mu \sigma \varrho=\omega \gamma \nu$.

(ii) $X_{2}=\varrho \varrho \sigma, X_{4}=\gamma \nu \omega, X_{5}=\xi \alpha \delta$.

(iii) $X_{2}=\tilde{X}_{2}+\lambda X_{2}^{m}, X_{4}=\tilde{X}_{4}+\lambda X_{4}^{m}, X_{5}=\tilde{X}_{5}+\lambda X_{5}^{m}$.

(iv) $X_{2}^{m}=\left(\tilde{X}_{2}\right)^{m}, X_{4}^{m}=\left(\tilde{X}_{4}\right)^{m}, X_{5}^{m}=\left(\tilde{X}_{5}\right)^{m}$. 
Proof The equalities in (i) and (ii) follow directly from the relations defining $\Lambda$. For (iii), observe that the vertices $2,4,5$ are in one orbit of the automorphism $\varphi$. Hence, it is enough to show that $X_{2}=\tilde{X}_{2}+\lambda X_{2}^{m}$. We have

$$
X_{2}=\varepsilon \xi \sigma=\rho \mu \sigma=\rho \omega \beta \text {. }
$$

Moreover

$$
\rho \omega \beta=\left(\varepsilon \eta+\lambda X_{2}^{m-1} \varepsilon \eta\right) \beta=\tilde{X}_{2}+\lambda X_{2}^{m-1} \varepsilon \eta \beta
$$

and

$$
X_{2}^{m-1} \varepsilon \eta \beta=X_{2}^{m-1} \varepsilon\left(\xi \sigma-\lambda X_{5}^{m-1} \eta \beta\right)=X_{2}^{m-1} \varepsilon \xi \sigma=X_{2}^{m} .
$$

The equalities in (iv) follow from the equalities in (iii) and the fact that $X_{2}^{m}, X_{4}^{m}, X_{5}^{m}$ are in the socle of $\Lambda$.

Lemma 4.4 For vertices $i \neq j$ in $Q$, any two paths of length 3 from $i$ to $j$ are equal and non-zero in $\Lambda$.

Proof Consider paths of length three between different vertices $i$ and $j$ in $Q$. Such paths only exist if the vertices are "opposite", and because of the automorphism $\varphi$, we may assume that $\{i, j\}=\{1,2\}$. Concerning paths from 1 to 2 we have

$$
\delta \eta \beta=\delta \xi \sigma-\lambda \delta X_{5}^{m-1} \eta \beta .
$$

Now, $\delta X_{5}=\delta \eta \gamma \delta=X_{1} \delta$ and therefore

$$
\delta X_{5}^{m-1} \eta \beta=X_{1}^{m-1} \delta \eta \beta=X_{s(\delta)}^{m-1} \delta f(\delta) g(f(\delta))=0 .
$$

With this, we have

$$
\delta \eta \beta=\delta \xi \sigma=v \mu \sigma=v \omega \beta,
$$

as required. A similar calculation shows that all paths from 2 to 1 of length three are equal in $\Lambda$.

Lemma 4.5 The following statements hold:

(i) For $4 \leq k \leq 3 m-1$, any two paths of length $k$ between two vertices in $Q$ are equal and non-zero in $\Lambda$.

(ii) For $k=3 m$, any path of length $k$ between two different vertices is zero in $\Lambda$.

(iii) For $k=3 m$, any cycle of length $k$ around a vertex $i$ is equal to $X_{i}^{m}$.

(iv) For $k>3 m$, any path of length $k$ is zero in $\Lambda$.

Proof For the following, we write $X_{i j}$ for a path of length three between vertices $i \neq j$. We first show that any two paths of length four between two fixed vertices are equal. For this, it suffices to consider paths starting at 1 and paths starting at 2 .

(i1) Paths from 1 of length four must end at vertex 5 or vertex 6 . Consider paths ending at 5. Such a path either ends with arrow $\delta$ or it ends with arrow $\varepsilon$. If it ends with $\delta$ then it is the product of a cyclic path of length three from 1 to 1 with $\delta$, hence by Lemma 4.3, is equal to $X_{1} \delta$. Similarly, any path of length four from 1 ending with $\varepsilon$ is the product of a path of length three from 1 to 2 with $\varepsilon$, hence is equal in $\Lambda$ to $X_{12} \varepsilon$. We must show that $X_{1} \delta=X_{12} \varepsilon$. We have

$$
X_{1} \delta=v \mu \alpha \delta=v \mu \sigma \varepsilon=X_{12} \varepsilon .
$$

Similarly, any path of length four from 1 to 6 ends with arrow $\rho$ or with arrow $v$, and one shows as above that all are equal in $\Lambda$. 
(i2) Consider paths of length four starting at vertex 2, any such path ends at vertex 6 or vertex 5. Consider paths ending at vertex 6 , the last arrow in such a path is $v$ or $\rho$. If it ends with $v$ then the path is of the form $X_{12} v$, and if it ends with $\rho$ then it is either $X_{2} \rho$, or it is $\tilde{X}_{2} \rho$. We have

$$
\tilde{X}_{2} \rho-\left(X_{2}-\lambda X_{2}^{m}\right) \rho=X_{2} \rho
$$

(noting that $X_{2}^{m}$ is in the right socle of $\Lambda$ ). Moreover,

$$
X_{2} \rho=\rho \omega \beta \rho=\rho \omega \gamma \nu=X_{12} \nu .
$$

For paths ending at vertex 5 the proof is similar.

We finish the proof of (i) by induction on $k$, using arguments as for the case $k=4$. Note that all paths of length $\leq 3 m-1$ in $Q$ are non-zero in $\Lambda$ since all zero relations of $\Lambda$ have length $3 m$ (and since the relations as listed are minimal).

We prove now the statements (ii) and (iii). It suffices again to consider paths starting at 1 and paths starting at 2 . A cyclic path starting at 1 of length $3 m$ is of the form $Y \gamma$ or $Y^{\prime} \alpha$, where $Y$ ends at vertex 4 and $Y^{\prime}$ ends at vertex 3. By part (i) we can take $Y=X_{1}^{m-1} \delta \eta$ and then $Y \gamma=X_{1}^{m}$. As well we can take $Y^{\prime}=X_{1}^{m-1} v \mu$ and get $Y^{\prime} \alpha=X_{1}^{m}$. Similarly, any path of length $3 m$ from 2 to 2 is equal to $X_{2}^{m}$. Now consider a path from vertex 1 of length $3 m$ which does not end at vertex 1, then it must end at vertex 2. It is of the form $Y \beta$ with $Y$ from 1 to 4 , or of the form $Y^{\prime} \sigma$ with $Y^{\prime}$ from 1 to 3. By part (i) we can take $Y=X_{1}^{m-1} \delta \eta$ and then

$$
Y \beta=X_{s(\delta)}^{m-1} \delta f(\delta) g(f(\delta))=0 .
$$

We also can take $Y^{\prime}=X_{1}^{m-1} v \mu$ and then again, by the defining relations, $Y^{\prime} \sigma=0$. Finally, consider a path from vertex 2 of length $3 m$ which does not end at vertex 2 , then it must end at vertex 1. Such a path is either of the form $Y \gamma$, or of the form $Y^{\prime} \alpha$, where $Y$ and $Y^{\prime}$ are paths of length $3 m-1$. We can take $Y=X_{2}^{m-1} \rho \omega$ and then $Y \gamma=0$, by the defining relations. Similarly, we can take $Y^{\prime}=X_{2}^{m-1} \varepsilon \xi$ and then $Y^{\prime} \alpha=0$, by the defining relations.

The statement (iv) follows because $X_{i}^{m}$ is in the right socle of $\Lambda$, for any vertex $i$ of $Q$.

We present now a basis of $\Lambda$ with good properties. We fix a vertex $i$, and define a basis $\mathcal{B}_{i}$ of $e_{i} \Lambda$ as follows. Choose a version of $X_{i}$, then suppose $X_{i}$ starts with $\tau$, then let $\bar{\tau}$ be the other arrow starting at $i$. Now let $\mathcal{B}_{i}:=$ the set of all initial subwords of $X_{i}^{m}$ together with the set

$$
\left\{X_{i}^{k} \bar{\tau}, X_{i}^{k} \bar{\tau} f(\bar{\tau}): 0 \leq k \leq m-1\right\} \cup\left\{X_{i}^{k} \tau f(\tau) g(f(\tau)): 0 \leq k<m-1\right\} .
$$

Then $\mathcal{B}_{i}$ is a basis for $e_{i} \Lambda$, and we take $\mathcal{B}:=\cup_{i \in Q_{0}} \mathcal{B}_{i}$. For each vertex $i$, let $\omega_{i}:=X_{i}^{m}$, this spans the socle of $e_{i} \Lambda$, by Lemma 4.5 , and it lies in $\mathcal{B}$. The basis $\mathcal{B}$ has the following properties:

(a) For each $k$ with $1 \leq k \leq 3 m-1$ the set $\mathcal{B}_{i}$ contains precisely two elements of length $k$. The end vertices are determined by the congruence of $k$ modulo 3 .

(b) Any path of length $k$ for $4 \leq k \leq 3 m-1$ is equal to precisely one basis element, as well any path of length three, except the cyclic paths between vertices $2,4,5$.

(c) The product of two elements $b, b^{\prime}$ from $\mathcal{B}$ is either zero, or is again an element in $\mathcal{B}$. It is non-zero if and only if $t(b)=s\left(b^{\prime}\right)$ and $b b^{\prime}$ has length $\leq 3 m$, and if the length is $3 m$ then $s(b)=t\left(b^{\prime}\right)$. (For this, note that the cyclic paths of length three through the vertices 2, 5, 4 are not products of basis elements.) 
(d) For each $b \in \mathcal{B}_{i}$ there is a unique $\hat{b} \in \mathcal{B}$ such that $b \hat{b}=\omega_{i}$ : Say $b=b e_{j}$ is of length $k$, then $\mathcal{B}_{j}$ must contain a unique element $\hat{b}$ of length $3 m-k$ and moreover which ends at $i$. This is seen by checking through each congruence. Then $b \hat{b}$ is a path of length $3 m$ from $i$ to $i$ and it must therefore be equal to $\omega_{i}$, by Lemma 4.5. It must be unique with $b b^{\prime}=\omega_{i}$ and $b^{\prime} \in \mathcal{B}$.

\section{Corollary 4.6 $\Lambda$ has dimension $36 m$.}

The next theorem completes the proof of Theorem 1 .

Theorem $4.7 \Lambda$ is a symmetric algebra.

Proof We use the above basis to define a symmetrizing bilinear form. If $b, b^{\prime} \in \mathcal{B}$, define

$$
\left(b, b^{\prime}\right):=\text { the coefficient of } \omega_{i} \text { when } b b^{\prime} \text { is expanded in terms of } \mathcal{B} \text {. }
$$

This extends to a bilinear form, and it is clearly associative. By (c) and (d) above, the Gram matrix of the bilinear form is non-singular, hence the form is non-degenerate. We show that the form is symmetric.

Let $b, b^{\prime} \in \mathcal{B}$, where $b=e_{i} b e_{j}$ and $b^{\prime}=e_{k} b^{\prime} e_{l}$. Then we have

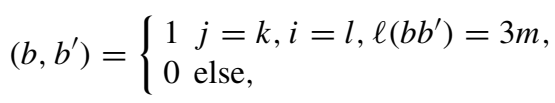

and $\left(b^{\prime}, b\right)$ is the same.

\section{Proof of Theorem 2}

Let $(Q, f)$ be the triangulation quiver associated to the tetrahedron. Then we have the involution $^{-}: Q_{1} \rightarrow Q_{1}$ on the set $Q_{1}$ of arrows of $Q$ which assigns to an arrow $\theta \in Q_{1}$ the arrow $\bar{\theta}$ with $s(\theta)=s(\bar{\theta})$ and $\theta \neq \bar{\theta}$. With this, we obtain another permutation $g: Q_{1} \rightarrow Q_{1}$ such that $g(\theta)=\overline{f(\theta)}$ for any $\theta \in Q_{1}$, as indicated in the introduction.

Let $m \geq 2$ be a natural number, $\lambda \in K$, and $\Lambda(m, \lambda)$ the associated higher tetrahedral algebra. We will prove first that $\Lambda(m, \lambda)$ is a tame algebra. We divide the proof into several steps.

Proposition 5.1 For each $\lambda \in K \backslash\{0\}, \Lambda(m, \lambda)$ degenerates to $\Lambda(m, 0)$.

Proof For each $t \in K$, consider the algebra $\Lambda(t)$ given by the quiver $Q$ and the relations:

$$
\begin{aligned}
& \gamma \delta=\beta \varepsilon+t \lambda(\beta \varrho \omega)^{m-1} \beta \varepsilon, \quad \delta \eta=\nu \omega, \quad \eta \gamma=\xi \alpha, \quad \nu \mu=\delta \xi, \\
& \varrho \omega=\varepsilon \eta+t \lambda(\varepsilon \xi \sigma)^{m-1} \varepsilon \eta, \quad \omega \beta=\mu \sigma, \quad \beta \varrho=\gamma \nu, \quad \mu \alpha=\omega \gamma, \\
& \xi \sigma=\eta \beta+t \lambda(\eta \gamma \delta)^{m-1} \eta \beta, \quad \sigma \varepsilon=\alpha \delta, \quad \varepsilon \xi=\varrho \mu, \quad \alpha \nu=\sigma \varrho, \\
& \left(\theta f(\theta) f^{2}(\theta)\right)^{m-1} \theta f(\theta) g(f(\theta))=0 \text { for any arrow } \theta \text { in } Q \text {. }
\end{aligned}
$$

Then $\Lambda(t), t \in K$, is an algebraic family in the variety $\operatorname{alg}_{d}(K)$, with $d=36 \mathrm{~m}$. Observe that $\Lambda(0) \cong \Lambda(m, 0)$ and $\Lambda(1) \cong \Lambda(m, \lambda)$. Fix $t \in K \backslash\{0\}$, and take an element $a_{t} \in K$ with $a_{t}^{3(m-1)}=t$. Then there is an isomorphism of algebras $\varphi_{t}: \Lambda(1) \rightarrow \Lambda(t)$ such that 
$\varphi_{t}(\theta)=a_{t} \theta$ for any arrow $\theta$ in $Q$. This shows that $\Lambda(t) \cong \Lambda(1)$ for all $t \in K \backslash\{0\}$. Then it follows from Proposition 2.2 that $\Lambda(m, \lambda)$ degenerates to $\Lambda(m, 0)=\Lambda(0)$.

Let $\Omega(m)$ be the algebra given by quiver $\Delta$ of the form

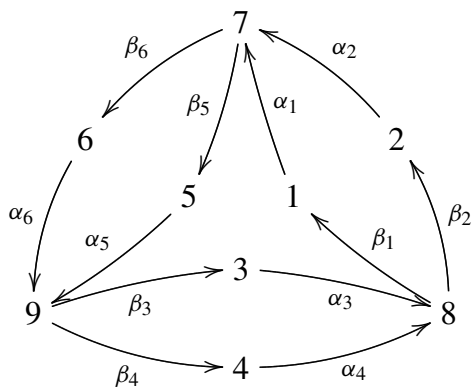

and the relations:

$$
\beta_{1} \alpha_{1}=\beta_{2} \alpha_{2}, \quad \beta_{3} \alpha_{3}=\beta_{4} \alpha_{4}, \quad \beta_{5} \alpha_{5}=\beta_{6} \alpha_{6},
$$

$\alpha_{1}\left(\beta_{5} \alpha_{5} \beta_{3} \alpha_{3} \beta_{1} \alpha_{1}\right)^{m-1} \beta_{5} \alpha_{5} \beta_{3} \alpha_{3} \beta_{2}=0, \quad \alpha_{2}\left(\beta_{6} \alpha_{6} \beta_{4} \alpha_{4} \beta_{2} \alpha_{2}\right)^{m-1} \beta_{6} \alpha_{6} \beta_{4} \alpha_{4} \beta_{1}=0$, $\alpha_{3}\left(\beta_{1} \alpha_{1} \beta_{5} \alpha_{5} \beta_{3} \alpha_{3}\right)^{m-1} \beta_{1} \alpha_{1} \beta_{5} \alpha_{5} \beta_{4}=0, \quad \alpha_{4}\left(\beta_{2} \alpha_{2} \beta_{6} \alpha_{6} \beta_{4} \alpha_{4}\right)^{m-1} \beta_{2} \alpha_{2} \beta_{6} \alpha_{6} \beta_{3}=0$, $\alpha_{5}\left(\beta_{3} \alpha_{3} \beta_{1} \alpha_{1} \beta_{5} \alpha_{5}\right)^{m-1} \beta_{3} \alpha_{3} \beta_{1} \alpha_{1} \beta_{6}=0, \quad \alpha_{6}\left(\beta_{4} \alpha_{4} \beta_{2} \alpha_{2} \beta_{6} \alpha_{6}\right)^{m-1} \beta_{4} \alpha_{4} \beta_{2} \alpha_{2} \beta_{5}=0$.

For each vertex $i$ of $\Delta$, we denote by $e_{i}$ the primitive idempotent of $\Omega(m)$ associated to $i$. Moreover, let $e=e_{1}+e_{2}+e_{3}+e_{4}+e_{5}+e_{6}$.

Lemma 5.2 The following statements hold:

(i) $\quad \Omega(m)$ is a finite-dimensional algebra with $\operatorname{dim}_{K} \Omega(m)=81 m+3$.

(ii) $\Lambda(m, 0)$ is isomorphic to the idempotent algebra $\mathrm{e} \Omega(m)$ e.

Proof (i) A direct checking shows that $\operatorname{dim}_{K} e_{i} \Omega(m)=9 m$ for $i \in\{1,2,3,4,5,6\}$, and $\operatorname{dim}_{K} e_{j} \Omega(m)=9 m+1$ for $j \in\{7,8,9\}$. Therefore, we obtain $\operatorname{dim}_{K} \Omega(m)=81 m+3$.

(ii) Consider the paths of length 2 in $\Delta$

$$
\begin{aligned}
& \delta=\alpha_{1} \beta_{5}, \quad v=\alpha_{1} \beta_{6}, \quad \varepsilon=\alpha_{2} \beta_{5}, \quad \varrho=\alpha_{2} \beta_{6}, \quad \alpha=\alpha_{3} \beta_{1}, \quad \sigma=\alpha_{3} \beta_{2}, \\
& \gamma=\alpha_{4} \beta_{1}, \quad \beta=\alpha_{4} \beta_{2}, \quad \xi=\alpha_{5} \beta_{3}, \quad \eta=\alpha_{5} \beta_{4}, \quad \mu=\alpha_{6} \beta_{3}, \quad \omega=\alpha_{6} \beta_{4} .
\end{aligned}
$$

Then these paths satisfy the relations defining the algebra $\Lambda(m, 0)$. Therefore, $e \Omega(m) e$ is isomorphic to $\Lambda(m, 0)$.

The algebra $\Omega(m)$ can be viewed as a blowup of the algebra $\Lambda(m, 0)$. The reason to consider it here is as follows. The higher tetrahedral algebras $\Lambda(m, \lambda)$ have no visible degenerations to special biserial alebras. But the algebra $\Omega(m)$ admits a degeneration to a special biserial algebra, as we will show below. Then Proposition 2.1 will imply that $\Omega(m)$ is a tame algebra, and consequently $\Lambda(m, 0)$ is a tame algebra (see [6, Theorem]). 
For each $t \in K$, let $\Sigma(m, t)$ be the algebra given by the quiver $\Sigma$ of the form

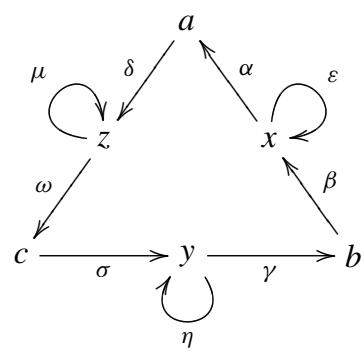

and the relations:

$$
\beta \alpha=0, \quad \sigma \gamma=0, \quad \delta \omega=0, \quad \varepsilon^{2}=t \varepsilon, \quad \eta^{2}=t \eta, \quad \mu^{2}=t \mu,
$$

$\begin{array}{ll}t(\alpha \delta \mu \omega \sigma \eta \gamma \beta \varepsilon)^{m}=\varepsilon(\alpha \delta \mu \omega \sigma \eta \gamma \beta \varepsilon)^{m}, & t(\varepsilon \alpha \delta \mu \omega \sigma \eta \gamma \beta)^{m}=(\varepsilon \alpha \delta \mu \omega \sigma \eta \gamma \beta)^{m} \varepsilon, \\ t(\gamma \beta \varepsilon \alpha \delta \mu \omega \sigma \eta)^{m}=\eta(\gamma \beta \varepsilon \alpha \delta \mu \omega \sigma \eta)^{m}, & t(\eta \gamma \beta \varepsilon \alpha \delta \mu \omega \sigma)^{m}=(\eta \gamma \beta \varepsilon \alpha \delta \mu \omega \sigma)^{m} \eta, \\ t(\omega \sigma \eta \gamma \beta \varepsilon \alpha \delta \mu)^{m}=\mu(\omega \sigma \eta \gamma \beta \varepsilon \alpha \delta \mu)^{m}, & t(\mu \omega \sigma \eta \gamma \beta \varepsilon \alpha \delta)^{m}=(\mu \omega \sigma \eta \gamma \beta \varepsilon \alpha \delta)^{m} \mu,\end{array}$

$$
\begin{aligned}
& (\alpha \delta \mu \omega \sigma \eta \gamma \beta \varepsilon)^{m}=(\varepsilon \alpha \delta \mu \omega \sigma \eta \gamma \beta)^{m}, \quad(\gamma \beta \varepsilon \alpha \delta \mu \omega \sigma \eta)^{m}=(\eta \gamma \beta \varepsilon \alpha \delta \mu \omega \sigma)^{m}, \\
& (\omega \sigma \eta \gamma \beta \varepsilon \alpha \delta \mu)^{m}=(\mu \omega \sigma \eta \gamma \beta \varepsilon \alpha \delta)^{m}, \\
& (\delta \mu \omega \sigma \eta \gamma \beta \varepsilon \alpha)^{m} \delta=0, \quad \alpha(\delta \mu \omega \sigma \eta \gamma \beta \varepsilon \alpha)^{m}=0, \quad(\beta \varepsilon \alpha \delta \mu \omega \sigma \eta \gamma)^{m} \beta=0, \\
& \gamma(\beta \varepsilon \alpha \delta \mu \omega \sigma \eta \gamma)^{m}=0, \quad(\sigma \eta \gamma \beta \varepsilon \alpha \delta \mu \omega)^{m} \sigma=0, \quad \omega(\sigma \eta \gamma \beta \varepsilon \alpha \delta \mu \omega)^{m}=0 .
\end{aligned}
$$

We note that for $t \in K \backslash\{0\}$ the relations (3) follow from the relations (2), and the relations (4) from the relations (1) and (2). For example, we have the equalities

$$
\begin{aligned}
t(\delta \mu \omega \sigma \eta \gamma \beta \varepsilon \alpha)^{m} \delta & =t \delta(\mu \omega \sigma \eta \gamma \beta \varepsilon \alpha \delta)^{m}=\delta(\mu \omega \sigma \eta \gamma \beta \varepsilon \alpha \delta)^{m} \mu \\
& =\delta \mu(\omega \sigma \eta \gamma \beta \varepsilon \alpha \delta \mu)^{m}=t \delta(\omega \sigma \eta \gamma \beta \varepsilon \alpha \delta \mu)^{m}=0,
\end{aligned}
$$

because $\delta \omega=0$, and hence $(\delta \mu \omega \sigma \eta \gamma \beta \varepsilon \alpha)^{m} \delta=0$, for $t \in K \backslash\{0\}$. For each vertex $i$ of $\Sigma$, we denote by $f_{i}$ the primitive idempotent of $\Sigma(m, t)$ associated to $i$.

Lemma 5.3 The following statements hold:

(i) For each $t \in K, \Sigma(m, t)$ is a finite-dimensional algebra with $\operatorname{dim}_{K} \Sigma(m, t)=81 m+$ 3.

(ii) $\Sigma(m, t) \cong \Sigma(m, 1)$ for any $t \in K \backslash\{0\}$.

(iii) $\Sigma(m, 0)$ is a special biserial algebra.

Proof (i) It follows from the relations defining $\Sigma(m, t)$ that $\operatorname{dim}_{K} f_{i} \Sigma(m, t)=9 m+1$ for $i \in\{a, b, c\}$, and $\operatorname{dim}_{K} f_{j} \Sigma(m, t)=18 m$ for $j \in\{x, y, z\}$. Hence, we obtain $\operatorname{dim}_{K} \Sigma(m, t)=81 m+3$.

(ii) Fix $t \in K \backslash\{0\}$, and take an element $b_{t} \in K$ with $b_{t}^{8}=t$. Then there exists an isomorphism of algebras $\psi_{t}: \Sigma(m, 1) \rightarrow \Sigma(m, t)$ such that $\psi_{t}(\varepsilon)=t^{-1} \varepsilon, \psi_{t}(\eta)=t^{-1} \eta$, $\psi_{t}(\mu)=t^{-1} \mu$, and $\psi_{t}(\theta)=b_{t} \theta$ for any arrow $\theta \in\{\alpha, \beta, \gamma, \sigma, \omega, \delta\}$.

(iii) Follows from the relations defining $\Sigma(m, 0)$.

Lemma 5.4 The algebras $\Omega(m)$ and $\Sigma(m, 1)$ are isomorphic. 
Proof We shall prove that there is a well defined isomorphism of algebras $\varphi: \Omega(m) \rightarrow$ $\Sigma(m, 1)$ such that

$$
\begin{array}{llll}
\varphi\left(e_{1}\right)=\varepsilon, & \varphi\left(e_{2}\right)=f_{x}-\varepsilon, & \varphi\left(e_{3}\right)=\eta, & \varphi\left(e_{4}\right)=f_{y}-\eta, \\
\varphi\left(e_{5}\right)=\mu, & \varphi\left(e_{6}\right)=f_{z}-\mu & \varphi\left(e_{7}\right)=f_{a}, & \varphi\left(e_{8}\right)=f_{b}, \quad \varphi\left(e_{9}\right)=f_{c}, \\
\varphi\left(\alpha_{1}\right)=\varepsilon \alpha, & \varphi\left(\alpha_{2}\right)=\alpha-\varepsilon \alpha, & \varphi\left(\beta_{1}\right)=\beta \varepsilon, & \varphi\left(\beta_{2}\right)=-\beta+\beta \varepsilon, \\
\varphi\left(\alpha_{3}\right)=\eta \gamma, & \varphi\left(\alpha_{4}\right)=\gamma-\eta \gamma, & \varphi\left(\beta_{3}\right)=\sigma \eta, & \varphi\left(\beta_{4}\right)=-\sigma+\sigma \eta, \\
\varphi\left(\alpha_{5}\right)=\mu \omega, & \varphi\left(\alpha_{6}\right)=\omega-\mu \omega, & \varphi\left(\beta_{5}\right)=\delta \mu, & \varphi\left(\beta_{6}\right)=-\delta+\delta \mu .
\end{array}
$$

Observe that

$$
\begin{aligned}
\varphi\left(e_{1}+e_{2}\right) & =f_{x}, & \varphi\left(e_{3}+e_{4}\right) & =f_{y}, & & \varphi\left(e_{5}+e_{6}\right)=f_{z}, \\
\varphi\left(\alpha_{1}+\alpha_{2}\right) & =\alpha, & \varphi\left(\alpha_{3}+\alpha_{4}\right) & =\gamma, & & \varphi\left(\alpha_{5}+\alpha_{6}\right)=\omega, \\
\varphi\left(\beta_{1}-\beta_{2}\right) & =\beta, & \varphi\left(\beta_{3}-\beta_{4}\right) & =\sigma, & & \varphi\left(\beta_{5}-\beta_{6}\right)=\delta .
\end{aligned}
$$

We have in $\Sigma(m, 1)$ the following equalities

$$
\begin{aligned}
\varphi\left(e_{1}^{2}\right) & =\varphi\left(e_{1}\right)=\varepsilon=\varepsilon^{2}=\varphi\left(e_{1}\right)^{2}, \\
\varphi\left(e_{2}^{2}\right) & =\varphi\left(e_{2}\right)=f_{x}-\varepsilon=\left(f_{x}-\varepsilon\right)^{2}=\varphi\left(e_{2}\right)^{2}, \\
\varphi\left(e_{3}^{2}\right) & =\varphi\left(e_{3}\right)=\eta=\eta^{2}=\varphi\left(e_{3}\right)^{2}, \\
\varphi\left(e_{4}^{2}\right) & =\varphi\left(e_{4}\right)=f_{y}-\eta=\left(f_{y}-\eta\right)^{2}=\varphi\left(e_{4}\right)^{2}, \\
\varphi\left(e_{5}^{2}\right) & =\varphi\left(e_{5}\right)=\mu=\mu^{2}=\varphi\left(e_{5}\right)^{2}, \\
\varphi\left(e_{6}^{2}\right) & =\varphi\left(e_{6}\right)=f_{z}-\mu=\left(f_{z}-\mu\right)^{2}=\varphi\left(e_{6}\right)^{2}, \\
\varphi\left(\beta_{1}\right) \varphi\left(\alpha_{1}\right) & =\beta \varepsilon^{2} \alpha=\beta \varepsilon \alpha=(-\beta+\beta \varepsilon)(\alpha-\varepsilon \alpha)=\varphi\left(\beta_{2}\right) \varphi\left(\alpha_{2}\right), \\
\varphi\left(\beta_{3}\right) \varphi\left(\alpha_{3}\right) & =\sigma \eta^{2} \gamma=\sigma \eta \gamma=(-\sigma+\sigma \eta)(\gamma-\eta \gamma)=\varphi\left(\beta_{4}\right) \varphi\left(\alpha_{4}\right), \\
\varphi\left(\beta_{5}\right) \varphi\left(\alpha_{5}\right) & =\delta \mu^{2} \omega=\delta \mu \omega=(-\delta+\delta \mu)(\omega-\mu \omega)=\varphi\left(\beta_{6}\right) \varphi\left(\alpha_{6}\right) .
\end{aligned}
$$

It remains to show that the six zero relations defining $\Omega(m)$ correspond via $\varphi$ to the six commutativity relations (2), with $t=1$, defining $\Sigma(m, 1)$. We will show this for the first two relations, because the proof for the other four is similar.

We have the equalities

$$
\begin{aligned}
& \varphi\left(\alpha_{1}\right)\left(\varphi\left(\beta_{5}\right) \varphi\left(\alpha_{5}\right) \varphi\left(\beta_{3}\right) \varphi\left(\alpha_{3}\right) \varphi\left(\beta_{1}\right) \varphi\left(\alpha_{1}\right)\right)^{m-1} \varphi\left(\beta_{5}\right) \varphi\left(\alpha_{5}\right) \varphi\left(\beta_{3}\right) \varphi\left(\alpha_{3}\right) \varphi\left(\beta_{2}\right) \\
& \quad=\varepsilon \alpha\left(\delta \mu^{2} \omega \sigma \eta^{2} \gamma \beta \varepsilon^{2} \alpha\right)^{m-1} \delta \mu^{2} \omega \sigma \eta^{2} \gamma(-\beta+\beta \varepsilon) \\
& \quad=-\varepsilon \alpha(\delta \mu \omega \sigma \eta \gamma \beta \varepsilon \alpha)^{m-1} \delta \mu \omega \sigma \eta \gamma \beta+\varepsilon \alpha(\delta \mu \omega \sigma \eta \gamma \beta \varepsilon \alpha)^{m-1} \delta \mu \omega \sigma \eta \gamma \beta \varepsilon \\
& \quad=-(\varepsilon \alpha \delta \mu \omega \sigma \eta \gamma \beta)^{m}+(\varepsilon \alpha \delta \mu \omega \sigma \eta \gamma \beta)^{m} \varepsilon=0, \\
& \varphi\left(\alpha_{2}\right)\left(\varphi\left(\beta_{6}\right) \varphi\left(\alpha_{6}\right) \varphi\left(\beta_{4}\right) \varphi\left(\alpha_{4}\right) \varphi\left(\beta_{2}\right) \varphi\left(\alpha_{2}\right)\right)^{m-1} \varphi\left(\beta_{6}\right) \varphi\left(\alpha_{6}\right) \varphi\left(\beta_{4}\right) \varphi\left(\alpha_{4}\right) \varphi\left(\beta_{1}\right) \\
& \quad=\varphi\left(\alpha_{2}\right)\left(\varphi\left(\beta_{5}\right) \varphi\left(\alpha_{5}\right) \varphi\left(\beta_{3}\right) \varphi\left(\alpha_{3}\right) \varphi\left(\beta_{1}\right) \varphi\left(\alpha_{1}\right)\right)^{m-1} \varphi\left(\beta_{5}\right) \varphi\left(\alpha_{5}\right) \varphi\left(\beta_{3}\right) \varphi\left(\alpha_{3}\right) \varphi\left(\beta_{1}\right) \\
& \quad=(\alpha-\varepsilon \alpha)(\delta \mu \omega \sigma \eta \gamma \beta \varepsilon \alpha)^{m-1} \delta \mu \omega \sigma \eta \gamma \beta \varepsilon \\
& \quad=(\alpha \delta \mu \omega \sigma \eta \gamma \beta \varepsilon)^{m}-\varepsilon(\alpha \delta \mu \omega \sigma \eta \gamma \beta \varepsilon)^{m}=0 .
\end{aligned}
$$

Corollary 5.5 The algebra $\Omega(m)$ degenerates to the special biserial algebra $\Sigma(m, 0)$. In particular, $\Omega(m)$ is a tame algebra.

Proof It follows from Lemmas 5.3 and 5.4 that $\Sigma(m, 1), t \in K$, is an algebraic family in the variety $\operatorname{alg}_{K}(d)$ with $d=81 m+3$ such that $\Sigma(m, 1) \cong \Sigma(m, 1) \cong \Omega(m)$ for any 
$t \in K \backslash\{0\}$ and $\Sigma(m, 0)$ is a special biserial algebra. Then it follows from Propositions 2.1 and 2.2 that $\Omega(m)$ is a tame algebra.

Proposition 5.6 For each $\lambda \in K, \Lambda(m, \lambda)$ is a tame algebra of non-polynomial growth.

Proof It follows from Lemma 5.2 (ii), Corollary 5.5 and [6, Theorem] that $\Lambda(m, 0)$ is a tame algebra. Then, applying Propositions 2.2 and 5.1, we conclue that $\Lambda(m, \lambda)$ is a tame algebra for any $\lambda \in K \backslash\{0\} . \Lambda=\Lambda(m, \lambda)$ for an arbitrary $\lambda \in K$. Consider now the quotient algebra $\Gamma$ of $\Lambda$ by the ideal generated by the arrows $\delta, v, \varepsilon, \varrho$. Then $\Gamma$ is the algebra given by the quiver

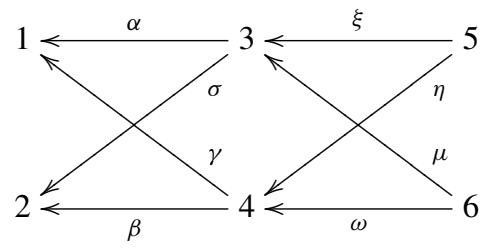

and the relations

$$
\omega \beta=\mu \sigma, \quad \eta \gamma=\xi \alpha, \quad \mu \alpha=\omega \gamma, \quad \xi \sigma=\eta \beta .
$$

Then $\Gamma$ is the tame minimal non-polynomial growth algebra (30) from [18]. Therefore, $\Lambda$ is of non-polynomial growth.

We end this section with a Galois covering interpretation of the singular higher tetrahedral algebras.

Let $m \geq 2$ be a natural number. We denote by $B(m)$ the fully commutative algebra of the following quiver
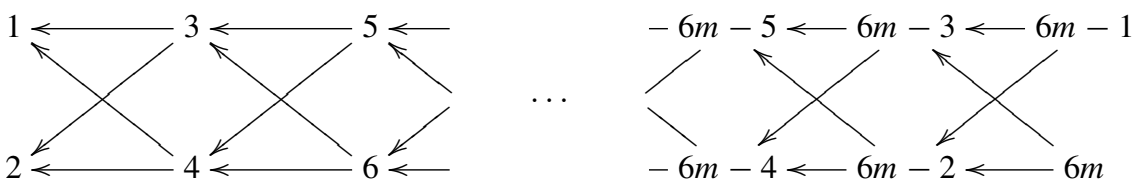

Consider the repetitive category $\widehat{B(m)}$ of $B(m)$. Then the Nakayama automorphism $v_{\widehat{B(m)}}$ of $\widehat{B(m)}$ admits an $m$-th root $\varphi_{m}$ such that $\left(\varphi_{m}\right)^{m}=v_{\widehat{B(m)}}$. Let $\Gamma(m)$ be the orbit algebra $\widehat{B(m)} /\left(\varphi_{m}\right)$ of $\widehat{B(m)}$ with respect to the infinite cyclic group $\left(\varphi_{m}\right)$ generated by $\varphi_{m}$ (see [24] for relevant definitions).

Then we obtain the following proposition.

Proposition 5.7 The algebras $\Lambda(m, 0)$ and $\Gamma(m)$ are isomorphic.

We would like to stress that, for any $\lambda \in K \backslash\{0\}$, the non-singular higher tetrahedral algebra $\Lambda(m, \lambda)$ is not the orbit algebra of the repetitive category of an algebra.

\section{Proof of Theorem 3}

Throughout this section, $\Lambda=\Lambda(m, \lambda)$, for some $m \geq 2$ and $\lambda \in K^{*}$. 
We show first that every simple $\Lambda$-module is periodic of period four. This will then tell us what the terms of a minimal projective bimodule resolution of $\Lambda$ must be (see Proposition 3.1). As for notation, we write $\Omega$ for syzygies of right $\Lambda$-modules, and we write $\Omega_{\Lambda^{e}}$ for syzygies of right $\Lambda^{e}$-modules $(\Lambda$ - $\Lambda$-bimodules $)$.

Proposition 6.1 Each simple $\Lambda$-module is periodic of period four. There is an exact sequence

$$
0 \rightarrow S_{i} \rightarrow P_{i} \rightarrow P_{x} \oplus P_{y} \rightarrow P_{j} \oplus P_{k} \rightarrow P_{i} \rightarrow S_{i} \rightarrow 0
$$

where the arrows adjacent to $i$ end at $j, k$ and start at $x, y$.

Proof The automorphism $\varphi$ of $\Lambda$ induces an equivalence of the module category $\bmod \Lambda$, with two orbits on simple modules. We only need to prove periodicity for one simple from each orbit. We will consider $S_{1}$ and $S_{4}$.

(1) We compute $\Omega^{2}\left(S_{1}\right)$ which we identify with the kernel of the map $d_{1}: P_{6} \oplus P_{5} \rightarrow P_{1}$ defined by

$$
d_{1}(a, b):=v a+\delta b,
$$

for $a \in P_{6}$ and $b \in P_{5}$. Since $\nu \omega=\delta \eta$ and $\nu \mu=\delta \xi$, the kernel contains the submodule generated by $\phi$ and $\psi$, where

$$
\phi=(-\omega, \eta) \text { and } \psi=(\mu,-\xi) .
$$

We will show that $\operatorname{Ker} d_{1}=\phi \Lambda+\psi \Lambda$. Since we have one inclusion, it suffices to show that both spaces have the same dimension, that is, we must show that $\phi \Lambda+\psi \Lambda$ has dimension $6 m+1$. We observe that $\phi \Lambda$ is isomorphic to $\Omega^{-1}\left(S_{4}\right)$ since $\omega, \eta$ are the arrows ending at vertex 4 . Similarly, $\psi \Lambda \cong \Omega^{-1}\left(S_{3}\right)$. In particular, $\operatorname{dim}_{K} \phi \Lambda=6 m-1=\operatorname{dim}_{K} \psi \Lambda$. It follows that we must show that $\operatorname{dim}_{K} \phi \Lambda \cap \psi \Lambda=6 m-3$, that is,

$$
\operatorname{dim}_{K} \phi \Lambda /(\phi \Lambda \cap \psi \Lambda)=2 .
$$

(1a) We identify the intersections of $\phi \Lambda$ and $\psi \Lambda$ with $0 \oplus P_{5}$. We claim that each of $\phi \Lambda \cap\left(0 \oplus P_{5}\right)$ and $\psi \Lambda \cap\left(0 \oplus P_{5}\right)$ is 1-dimensional, spanned by $\left(0, X_{5}^{m}\right)$. Indeed, suppose $\phi p=(0, z)$ for some $p \in \Lambda$ and $0 \neq z$. We may assume that $p$ is a monomial in the arrows. To have $\omega p=0$ the monomial $p$ must have length $\geq 3 m-1$. To have $\omega p=0$ and $\eta p=z \neq 0$, we must have that $p$ has length $3 m-1$ and ends at vertex 4 , and then $\eta p=X_{5}^{m}$. For the converse, take $p=X_{4}^{m-1} \gamma \delta$. Similarly one proves the second statement.

(1b) We claim that $\phi J^{2}+\phi \gamma K$ is contained in the intersection $\phi \Lambda \cap \psi \Lambda$. Namely, we have $\phi \gamma=-\psi \alpha$, by the relations. Next, we have

$$
\phi \beta=(-\omega \beta, \eta \beta)=-\psi \sigma-\left(0, \lambda X_{5}^{m-1} \eta \beta\right) .
$$

Hence $\phi \beta \varepsilon=\psi \sigma \varepsilon-\left(0, \lambda X_{5}^{m}\right)$ (using Lemmas $\left.4.3,4.4,4.5\right)$. By (1a) above, this belongs to the intersection and it follows from these that $\phi J^{2} \subseteq \phi \Lambda \cap \psi \Lambda$. We note that if $\lambda=0$, then $\Omega^{2}\left(S_{i}\right)$ has more than two minimal generators, and hence $S_{i}$ is not periodic of period 4 .

(1c) Note that $\phi J^{2}+\phi \gamma K$ has dimension $6 m-3$. We have the chain of submodules

$$
\phi J^{2}+\phi \gamma K \subseteq \phi \Lambda \cap \psi \Lambda \subseteq \phi \Lambda,
$$

and the quotient $\phi \Lambda /\left(\phi J^{2}+\phi \gamma K\right)$ is spanned by the cosets of $\phi$ and $\phi \beta$.

Assume for a contradiction that $\phi \beta \in \phi \Lambda \cap \psi \Lambda$. Then, by (*), we have $\left(0, X_{5}^{m-1} \eta \beta\right) \in$ $\psi \Lambda$, but this contradicts (1a). So $\phi \beta$ is not in the intersection, and therefore the dimension of $\phi \Lambda /(\phi \Lambda \cap \psi \Lambda)$ is 2 , as required. 

by

(1d) Now it is easy to see that $S_{1}$ has period four. Namely, define $d_{2}: P_{4} \oplus P_{3} \rightarrow \Omega^{2}\left(S_{1}\right)$

$$
d_{2}(u, v):=\phi u+\psi v,
$$

for $u \in P_{4}$ and $v \in P_{3}$. The kernel of $d_{2}$, that is, $\Omega^{3}\left(S_{1}\right)$ has dimension $2(6 m)-(6 m+1)=$ $6 m-1$. We have seen that $\phi \gamma=-\psi \alpha$, and therefore $(\gamma, \alpha) \Lambda \subseteq \operatorname{Ker}\left(d_{2}\right)$. This submodule is isomorphic to $\Omega^{-1}\left(S_{1}\right)$ and has dimension $6 m-1$. We deduce that

$$
\Omega^{-1}\left(S_{1}\right) \cong(\gamma, \alpha) \Lambda \cong \Omega^{3}\left(S_{1}\right) .
$$

So $S_{1}$ is periodic of period dividing 4 , and then equal to 4 .

(2) We compute $\Omega^{2}\left(S_{4}\right)$, which we identify with the kernel of $d_{1}: P_{1} \oplus P_{2} \rightarrow P_{4}$ defined as

$$
d_{1}(w, z)=\gamma w+\beta z,
$$

for $w \in P_{1}$ and $z \in P_{2}$. This is analogous to (1), there is only a small difference in the formulae. Using the relations, the kernel of $d_{1}$ contains $\phi$ and $\psi$, where

$$
\phi=\left(\delta,-\varepsilon-\lambda(\rho \omega \beta)^{m-1} \varepsilon\right) \text { and } \psi=(-v, \rho) .
$$

By the same arguments as in (1), to prove that $\operatorname{Ker}\left(d_{1}\right)=\phi \Lambda+\psi \Lambda$, we must show that $\operatorname{dim}_{K} \phi \Lambda /(\phi \Lambda \cap \psi \Lambda)=2$. We have $\phi \eta=-\psi \omega$, which is in the intersection, and we have

$$
\phi \xi=-\psi \mu-\left(0, \lambda X_{2}^{m-1} \varepsilon \xi\right) .
$$

As before one shows that $\phi J^{2}=\psi J^{2}$ and hence is in the intersection. Suppose $\phi \xi$ is in the intersection. Then it follows that $\left(0,-\lambda X_{2}^{m-1} \varepsilon \xi\right)$ is in $\psi \Lambda$, which is a contradiction to the analog of (1a). It follows that $\phi \Lambda /(\phi \Lambda \cap \psi \Lambda)$ is 2-dimensional. Then as in (1d) one concludes that $S_{4}$ has $\Omega$-period four.

We use the notation as in Section 3, in particular the description of $\mathbb{P}_{0}$ and $\mathbb{P}_{1}$. For the higher tetrahedral algebra, we need to specify $\mathbb{P}_{2}$, which has generators corresponding to the minimal relations involving paths of length two. Each of these minimal relations has a term $\theta f(\theta)$ for $\theta$ an arrow, and this gives a bijection between arrows and minimal relations involving paths of length two. So we take

$$
\mathbb{P}_{2}:=\oplus_{\theta \in Q_{1}} \Lambda\left(e_{s(\theta)} \otimes e_{t(f(\theta))}\right) \Lambda .
$$

We may denote the minimal relation with term $\theta f(\theta)$ by $\mu_{\theta}$. Then the definition of $R$ in Section 3 specializes to

$$
R: \mathbb{P}_{2} \rightarrow \mathbb{P}_{1}, \quad R\left(e_{s(\theta)} \otimes e_{t(f(\theta))}\right):=\pi\left(\mu_{\theta}\right) .
$$

Lemma 6.2 The homomorphism $R: \mathbb{P}_{2} \rightarrow \mathbb{P}_{1}$ induces a projective cover of $\Omega_{\Lambda^{e}}^{2}(\Lambda)$ in $\bmod \Lambda^{e}$. In particular, $\Omega_{\Lambda^{e}}^{3}(\Lambda)=\operatorname{Ker} R$.

Proof This is similar as that of Lemma 7.2 of [11], and uses Lemma 3.4.

By Propositions 3.1 and 6.1, we can take $\mathbb{P}_{3}=\oplus_{i \in Q_{0}} \Lambda\left(e_{i} \otimes e_{i}\right) \Lambda$. For each vertex $i$ of $Q$, we define an element $\psi_{i}$ as follows. Let $\tau, \bar{\tau}$ be the arrows starting at $i$, and let $\theta, \bar{\theta}$ be the arrows ending at $i$. Set

$$
\psi_{i}:=\left(e_{i} \otimes e_{t(\theta)}\right) \theta+\left(e_{i} \otimes e_{t(\bar{\theta})}\right) \bar{\theta}-\tau\left(e_{t(\tau)} \otimes e_{i}\right)-\bar{\tau}\left(e_{t(\bar{\tau})} \otimes e_{i}\right) .
$$

Then we define a $\Lambda^{e}$-module homomorphism $S: \mathbb{P}_{3} \rightarrow \mathbb{P}_{2}$ by

$$
S\left(e_{i} \otimes e_{i}\right):=\psi_{i} \text {, for } i \in Q_{0} .
$$


Lemma 6.3 The homomorphism $S: \mathbb{P}_{3} \rightarrow \mathbb{P}_{2}$ induces a projective cover of $\Omega_{\Lambda^{e}}^{3}(\Lambda)$ in $\bmod \Lambda^{e}$. In particular, we have $\Omega_{\Lambda^{e}}^{4}(\Lambda)=\operatorname{Ker}(S)$.

Proof We know that the kernel of $R$ is $\Omega_{\Lambda^{e}}^{3}(\Lambda)$, and we know that it has minimal generators corresponding to the vertices of $Q$. As well, from the definition, the element $\psi_{i}$ does not lie in $\left(\operatorname{rad} \mathbb{P}_{2}\right)^{2}$. Therefore, it is enough to show that $R\left(\psi_{i}\right)=0$ for all $i$.

The algebra automorphism $\varphi$ of $\Lambda$ defined in Section 4, extends to an automorphism of $\Lambda^{e}$. One checks that it commutes with the map $R$ and that it takes $\psi_{i}$ to $\psi_{\varphi(i)}$. So it is enough to take $i=1$ and $i=4$.

(1) We compute $R\left(\psi_{1}\right)$. This is equal to

$$
\begin{aligned}
R( & \left.\left(e_{1} \otimes e_{4}\right) \gamma+\left(e_{1} \otimes e_{3}\right) \alpha-v\left(e_{6} \otimes e_{1}\right)-\delta\left(e_{5} \otimes e_{1}\right)\right) \\
= & \pi(\delta \eta-v \omega) \gamma+\pi(v \mu-\delta \xi) \alpha-v(\pi(\mu \alpha-\omega \gamma))-\delta(\pi(\eta \gamma-\xi \alpha)) \\
= & \left(e_{1} \otimes \eta \gamma+\delta \otimes \gamma-e_{1} \otimes \omega \gamma-v \otimes \gamma\right) \\
& +\left(e_{1} \otimes \mu \alpha+v \otimes \alpha-e_{1} \otimes \xi \alpha-\delta \otimes \alpha\right) \\
& -\left(v \otimes \alpha+v \mu \otimes e_{1}-v \otimes \gamma-v \omega \otimes e_{1}\right) \\
& -\left(\delta \otimes \gamma+\delta \eta \otimes e_{1}-\delta \otimes \alpha-\delta \xi \otimes e_{1}\right) .
\end{aligned}
$$

The terms of the form $\alpha_{1} \otimes \alpha_{2}$ for $\alpha_{i}$ arrows, cancel. The terms in $\left(e_{1} \otimes e_{5}\right) \Lambda$ are

$$
\left.e_{1} \otimes \eta \gamma-e_{1} \otimes \xi \alpha\right)=e_{1} \otimes(\eta \gamma-\xi \alpha)=0 .
$$

Similarly, there are two terms in $\left(e_{1} \otimes e_{6}\right) \Lambda$ and two terms in $\Lambda\left(e_{4} \otimes e_{1}\right)$ and two terms in $\Lambda\left(e_{3} \otimes e_{1}\right)$, and they all cancel. Hence $R\left(\psi_{1}\right)=0$.

(2) We compute $R\left(\psi_{4}\right)$. This is equal to

$$
\begin{gathered}
R\left(\left(e_{4} \otimes e_{5}\right) \eta+\left(e_{4} \otimes e_{6}\right) \omega-\gamma\left(e_{1} \otimes e_{4}\right)-\beta\left(e_{2} \otimes e_{4}\right)\right) \\
=\pi\left(\gamma \delta-\beta \varepsilon-\lambda X_{4}^{m-1} \beta \varepsilon\right) \eta+\pi(\beta \rho-\gamma \nu) \omega \\
\quad-\gamma(\pi(\delta \eta-v \omega))-\beta\left(\pi\left(\rho \omega-\varepsilon \eta-\lambda X_{2}^{m-1} \varepsilon \eta\right)\right) .
\end{gathered}
$$

We must choose a version of $X_{4}$ and of $X_{2}$. It is natural to take $X_{4}=\beta \rho \omega$ and $X_{2}=\rho \omega \beta$. We continue the calculation. With this, $\left(^{*}\right)$ is equal to

$$
\begin{aligned}
& \left(e_{4} \otimes \delta \eta+\gamma \otimes \eta-e_{4} \otimes \varepsilon \eta-\beta \otimes \eta\right)-\lambda \pi\left((\beta \rho \omega)^{m-1} \beta \varepsilon\right) \eta \\
& \quad+\left(e_{4} \otimes \rho \omega+\beta \otimes \omega-e_{4} \otimes \nu \omega-\gamma \otimes \omega\right) \\
& \quad-\left(\gamma \otimes \eta+\gamma \delta \otimes e_{4}-\gamma \otimes \omega-\gamma \nu \otimes e_{4}\right) \\
& \quad-\left(\beta \otimes \omega+\beta \rho \otimes e_{4}-\beta \otimes \eta-\beta \varepsilon \otimes e_{4}\right)+\lambda \beta\left(\pi\left((\rho \omega \beta)^{m-1} \varepsilon \eta\right)\right) .
\end{aligned}
$$

The terms of the form $\alpha_{1} \otimes \alpha_{2}$ with $\alpha_{i}$ arrows all cancel. Using the relations $\delta \eta=\nu \omega$ and $\beta \rho=\gamma \nu$, four of the other terms cancel. This leaves

$$
-e_{4} \otimes \varepsilon \eta+e_{4} \otimes \rho \omega-\lambda \pi\left((\beta \rho \omega)^{m-1} \beta \varepsilon\right) \eta-\gamma \delta \otimes e_{4}+\beta \varepsilon \otimes e_{4}+\lambda \beta \pi\left((\rho \omega \beta)^{m-1} \varepsilon \eta\right) .
$$

The first two terms combine, and the fourth and fifth term combine, and we can rewrite the expression as

$$
\lambda\left(e_{4} \otimes X_{2}^{m-1} \varepsilon \eta\right)-\lambda \pi\left((\beta \rho \omega)^{m-1} \beta \varepsilon\right) \eta-\lambda\left(X_{4}^{m-1} \beta \varepsilon \otimes e_{4}\right)+\lambda \beta \pi\left((\rho \omega \beta)^{m-1} \varepsilon \eta\right) .
$$

Now we combine the second and fourth term of (**), and we expand both. All terms except the ones $-\otimes e_{4}$ and $e_{4} \otimes-$ cancel, and we are left with

$$
\lambda\left((\beta \rho \omega)^{m-1} \beta \varepsilon \otimes e_{4}-e_{4} \otimes(\rho \omega \beta)^{m-1} \varepsilon \eta\right) .
$$

The first term of $(* * *)$ is the negative of the third term in $(* *)$ since $\beta \rho \omega=X_{4}$. The second term of $(* * *)$ is the negative of the first term of (**) since $\rho \omega \beta=X_{2}$. Hence, everything cancels and $R\left(\psi_{4}\right)=0$, as required. 
Theorem 6.4 There is an isomorphism $\Omega_{\Lambda^{e}}^{4}(\Lambda) \cong \Lambda$ in $\bmod \Lambda^{e}$.

Proof This is similar as in the proof of Theorem 7.4 in [11]. We have defined a symmetrizing bilinear form of $\Lambda$ in the proof of Theorem 4.7. We define elements $\xi_{i} \in \mathbb{P}_{3}$ by

$$
\xi_{i}=\sum_{b \in \mathcal{B}_{i}} b \otimes b^{*}
$$

where $\left\{b^{*}: b \in \mathcal{B}\right\}$ is the dual basis corresponding to $\mathcal{B}$, defined by $(-,-)$. As in [11], it follows that the map

$$
\theta: \Lambda \rightarrow \mathbb{P}_{3} \text {, with } \theta\left(e_{i}\right)=\xi_{i} \text { for all } i \in Q_{0},
$$

is a monomorphism of $\Lambda-\Lambda$-bimodules. Moreover, one shows that $S\left(\xi_{i}\right)=0$, exactly as in [11], and hence the image of $\theta$ is contained in $\Omega_{\Lambda^{e}}^{4}(\Lambda)=\operatorname{Ker} S$ (Lemma 6.3). This only uses general properties of the dual basis and no details on a specific algebra. Furthermore, $\Omega_{\Lambda^{e}}^{4}(\Lambda)$ is free of rank 1 as a left or right $\Lambda$-module. Namely, by Lemmas 3.3, 6.2, and 6.3, we have the exact sequence of bimodules

$$
0 \rightarrow \Omega_{\Lambda^{e}}^{4}(\Lambda) \rightarrow \mathbb{P}_{3} \stackrel{S}{\rightarrow} \mathbb{P}_{2} \stackrel{R}{\rightarrow} \mathbb{P}_{1} \stackrel{d}{\rightarrow} \mathbb{P}_{0} \rightarrow \Lambda \rightarrow 0
$$

We have $\mathbb{P}_{0} \cong \mathbb{P}_{3}$, and moreover $\mathbb{P}_{1}$ and $\mathbb{P}_{2}$ have obviously the same rank as free $\Lambda$-modules on each side. By the exactness, it follows that $\Lambda$ and $\Omega_{\Lambda^{e}}^{4}(\Lambda)$ have the same rank. Therefore, the map $\theta$ gives an isomorphism of $\Lambda$ with $\Omega_{\Lambda^{e}}^{4}(\Lambda)$.

Alternatively, for the last step one may apply [14] to show that $\Omega_{\Lambda^{e}}^{4}(\Lambda)$ must be isomorphic to ${ }_{1} \Lambda_{\sigma}$ for some algebra automorphism $\sigma$, and therefore has rank 1 on each side.

Theorem 3 follows from Proposition 6.1, Theorem 6.4, and the following proposition.

Proposition 6.5 Let $A=\Lambda(m, 0)$. Then $\bmod A$ does not admit a periodic simple module.

Proof Take $i \in\{1,3,5\}$. Observe that, for the indecomposable projective $A$-modules $P_{i}=$ $e_{i} A$ and $P_{i+1}=e_{i+1} A$, we have $\operatorname{rad} P_{i} / \operatorname{soc} P_{i} \cong \operatorname{rad} P_{i+1} / \operatorname{soc} P_{i+1}$ in $\bmod A$. Then, by general theory, $P_{i} / \operatorname{soc} P_{i}$ and $P_{i+1} /$ soc $P_{i+1}$ are not in stable tubes of the stable AuslanderReiten quiver $\Gamma_{A}^{S}$ of $A$. Since $A$ is a symmetric algebra, we conclude that $S_{i}$ and $S_{i+1}$ are not periodic modules.

Acknowledgements The research was done during the visit of the first named author at the Faculty of Mathematics and Computer Sciences in Toruń (June 2017).

Open Access This article is distributed under the terms of the Creative Commons Attribution 4.0 International License (http://creativecommons.org/licenses/by/4.0/), which permits unrestricted use, distribution, and reproduction in any medium, provided you give appropriate credit to the original author(s) and the source, provide a link to the Creative Commons license, and indicate if changes were made.

\section{References}

1. Assem, I., Simson, D., Skowroński, A.: Elements of the Representation Theory of Associative Algebras 1: Techniques of Representation Theory London Mathematical Society Student Texts, vol. 65. Cambridge University Press, Cambridge (2006) 
2. Białkowski, J., Erdmann, K., Skowroński, A.: Periodicity of self-injective algebras of polynomial growth. J. Algebra 443, 200-269 (2015)

3. Butler, M.C.R., Ringel, C.M.: Auslander-Reiten sequences with few middle terms and applications to string algebras and bocses. Comm. Algebra 15, 145-179 (1987)

4. Crawley-Boevey, W.: On tame algebras and bocses. Proc. London Math. Soc. 56, 451-483 (1988)

5. Crawley-Boevey, W.: Tameness of biserial algebras. Arch. Math. (Basel) 65, 399-407 (1995)

6. Dowbor, P., Skowroński, A.: On the representation type of locally bounded categories. Tsukuba J. Math. 10, 63-72 (1986)

7. Dowbor, P., Skowroński, A.: Galois coverings of representation-infinite algebras. Comment. Math. Helv. 62, 311-337 (1987)

8. Drozd, Y.A.: Tame and wild matrix problems. In: Representation Theory II, Lecture Notes in Math., vol. 832, pp. 242-258. Springer, Berlin-Heidelberg (1980)

9. Dugas, A.: Periodic resolutions and self-injective algebras of finite type. J. Pure Appl. Algebra 214, 990-1000 (2010)

10. Erdmann, K., Skowroński, A.: Periodic algebras. In: Trends in Representation Theory of Algebras and Related Topics, Europ. Math. Soc. Series Congress Reports, pp. 201-251. European Math. Soc., Zürich (2008)

11. Erdmann, K., Skowroński, A.: Weighted surface algebras. arXiv:1703.02346 (2017)

12. Erdmann, K., Skowroński, A.: Algebras of generalized quaternion type. arXiv:1710.09640 (2017)

13. Geiss, C.: On degenerations of tame and wild algebras. Arch. Math. (Basel) 64, 11-16 (1995)

14. Green, E.L., Snashall, N., Solberg, Ø.: The Hochschild cohomology ring of a selfinjective algebra of finite representation type. Proc. Amer. Math. Soc. 131, 3387-3393 (2003)

15. Happel, D.: Triangulated Categories in the Representation Theory of Finite-Dimensional Algebras, London Math. Soc. Lect. Note Ser., vol. 119. Cambridge University Press, Cambridge (1988)

16. Happel, D.: Hochschild cohomology of finite-dimensional algebras. In: Séminaire d'Algèbre Paul Dubreil Et Marie-Paul Malliavin, Lecture Notes in Math., vol. 1404, pp. 108-126. Springer, BerlinHeidelberg (1989)

17. Kraft, H.: Geometric Methods in Representation Theory. In: Representations of Algebras, Lecture Notes in Math., vol. 944, pp. 180-258. Springer, Berlin-Heidelberg (1982)

18. Nörenberg, R., Skowroński, A.: Tame minimal non-polynomial growth simply connected algebras. Colloq. Math. 73, 301-330 (1997)

19. Pogorzały, Z., Skowroński, A.: Selfinjective biserial standard algebras. J. Algebra 138, 491-504 (1991)

20. Rickard, J.: Derived equivalences as derived functors. J. London Math. Soc. 43, 37-48 (1991)

21. Ringel, C.M.: Tame algebras and integral quadratic forms. In: Lecture Notes in Math., vol. 1099. Springer, Berlin-Heidelberg (1984)

22. Simson, D., Skowroński, A.: Elements of the Representation Theory of Associative Algebras 3: Representation-Infinite Tilted Algebras London Mathematical Society Student Texts, vol. 72. Cambridge University Press, Cambridge (2007)

23. Skowroński, A.: Selfinjective algebras of polynomial growth. Math. Ann. 285, 177-199 (1989)

24. Skowroński, A.: Selfinjective algebras: finite and tame type. In: Trends in Representation Theory of Algebras and Related Topics, Contemp. Math., vol. 406, pp. 169-238. Amer. Math. Soc., Providence (2006)

25. Skowroński, A., Waschbüsch, J.: Representation-finite biserial algebras. J. Reine Angew. Math. 345, 172-181 (1983)

26. Skowroński, A., Yamagata, K.: Frobenius Algebras I: Basic Representation Theory, European Mathematical Society Textbooks in Mathematics. European Math. Soc. Publ. House, Zürich (2011)

27. Wald, B., Waschbüsch, J.: Tame biserial algebras. J. Algebra 95, 480-500 (1985) 